\title{
FXR1P But Not FMRP Regulates the Levels of Mammalian Brain-Specific microRNA-9 and microRNA-124
}

\author{
Xia-Lian Xu, ${ }^{1}$ Ruiting Zong, ${ }^{2}$ Zhaodong Li, ${ }^{3}$ Md Helal Uddin Biswas, ${ }^{3}$ Zhe Fang, ${ }^{2}$ David L. Nelson, ${ }^{2}$ and Fen-Biao Gao ${ }^{3}$ \\ ${ }^{1}$ Gladstone Institute of Neurological Disease, San Francisco, California 94158, ${ }^{2}$ Department of Molecular and Human Genetics, Baylor College of Medicine, \\ Houston, Texas 77030, and ${ }^{3}$ Department of Neurology, University of Massachusetts Medical School, Worcester, Massachusetts 01605
}

Mammalian brain-specific miR-9 and miR-124 have been implicated in several aspects of neuronal development and function. However, it is not known how their expression levels are regulated in vivo. We found that the levels of miR-9 and miR-124 are regulated by FXR1P but not by the loss of FXR2P or FMRP in vivo, a mouse model of fragile X syndrome. Surprisingly, the levels of miR-9 and miR-124 are elevated in $f m r 1 / f x r 2$ double-knock-out mice, in part reflecting posttranscriptional upregulation of FXR1P. Indeed, FXR1P is required for efficient processing of pre-miR-9 and pre-miR-124 in vitro and forms a complex with Dicer and pre-miRNAs. These findings reveal differential roles of FMRP family proteins in controlling the expression levels of brain-specific miRNAs.

\section{Introduction}

MicroRNAs (miRNAs) are critical for posttranscriptional regulation of many aspects of neuronal development and function (Coolen and Bally-Cuif, 2009; Schratt, 2009). Among them, the cellular context-dependent functions of miR-9 and miR-124 have been extensively studied (Gao, 2010). Both miRNAs are specifically expressed in mammalian brains including postmitotic neurons (Deo et al., 2006), and their respective nucleotide sequences are evolutionarily conserved. The levels of these neuronal miRNAs are tightly regulated both spatially and temporally; however, the underlying mechanisms remain to be fully understood.

Mature miR-9 and miR-124 are each produced from three precursor miRNAs (pre-miRNAs) located on separate chromosomes, and each pre-miRNA is transcribed at different levels. For instance, pre-miR-9-2 is predominant in the mouse embryonic brain (Shibata et al., 2008) and human neural progenitor cells (Delaloy et al., 2010). miRNAs levels are also affected by the efficiency of processing by the Dicer and associated proteins, and from the primary miRNA transcripts (pri-miRNAs) to pre-miRNAs by the Microprocessor complex (Newman and Hammond, 2010). Although some previously characterized RNA-binding proteins have been implicated in these processes, such as hRNP proteins (Gregory et al., 2004; Michlewski et al., 2008), the precise roles of other RNA-binding proteins in regulating miRNA abundance are unclear. Moreover, miRNAs in neurons are much less stable than that in non-neuronal cells and can be regulated by neuronal activity (Krol et al., 2010).

Received June 6, 2011; revised July 8, 2011; accepted July 14, 2011.

Author contributions: F.-B.G. designed research; X.-L.X., R.Z., Z.L., H.U.B., and Z.F. performed research; X.-L.X. and F.-B.G. analyzed data; X.-L.X., Z.L., H.U.B., D.L.N., and F.-B.G. wrote the paper.

This work was supported by the NIH (F.-B.G., D.L.N.). We thank L. Liu for help with mouse genotyping, S. Ordway for editorial assistance, A. Wilson for help with graphics, and laboratory members for discussions.

Correspondence should be addressed to Fen-Biao Gao, Department of Neurology, University of Massachusetts Medical School, Worcester, MA 01605. E-mail: fen-bia0.ga0@umassmed.edu.

DOI:10.1523/JNEUROSCI.2827-11.2011

Copyright $\odot 2011$ the authors $\quad 0270-6474 / 11 / 3113705-05 \$ 15.00 / 0$
Drosophila fragile $\mathrm{X}$ mental retardation protein 1 (dFMR1) is associated with the miRNAs pathway (Caudy et al., 2002; Ishizuka et al., 2002). In $d f m r 1$ mutant flies, the steady-state level of miR-124 is reduced, and the effect of miR-124 overexpression on dendritic branching is attenuated (Xu et al., 2008). In mammals, FMRP is homologous to two closely related proteins, FXR1P and FXR2P (Zhang et al., 1995). All three proteins are coexpressed in virtually all differentiated neurons (Tamanini et al., 1997). Loss of FMRP in humans causes fragile X syndrome-the most common form of inherited intellectual disability. The molecular functions of this protein in neuronal development and synaptic function are incompletely understood (Waung and Huber, 2009). Among its roles, FMRP is required for miR-125b regulation of NR2A expression and spine morphology (Edbauer et al., 2010). However, it is not known whether FMRP family proteins affect the expression levels of brain-specific miRNAs. To address this issue, in this study, we used single or double knock-out mice to examine the specific roles of FMRP, FXR1P, and FXR2P in regulating miR-9 and miR-124 levels in the mammalian brain.

\section{Materials and Methods}

Mouse strains and tissue collection. Control wild-type (WT) and homozygous fmr $1 \mathrm{KO}$ mice of either sex were from The Jackson Laboratory. WT, $f x r 2$, fmrl/fxr 2 double $\mathrm{KO}$, and $f x r 1 \mathrm{KO}$ mice of either sex and frozen whole brain and muscle tissues from the mice were from the laboratory of D. L. Nelson. Unless indicated otherwise, experiments were conducted in adult mice of either sex at $\sim 2$ months of age. Mice were anesthetized and killed by cervical dislocation, and specified tissues were immediately removed for RNA isolation or protein extraction.

RNA isolation and quantitative real-time PCR ( $q R T-P C R)$ analysis. Total RNAs were isolated from mouse tissues or cultured cells with Trizol (Invitrogen) and purified with RNeasy Mini or miRNeasy kits (Qiagen). Mature miRNAs were detected with a TaqMan miRNA reverse transcription kit followed by real-time PCR (Applied Biosystems); expression levels were normalized to U6 RNA levels. mRNA levels were measured by qRT-PCR as described previously (Xu et al., 2008) and normalized to GAPDH expression. The primers for measuring miR-124 were as follows. miR-124-1-forward: CGGGTAGCAGGCTTCTGAGT; miR-124-1-re- 
verse: AAACCCCTC TCTGTCGGTAGCT; miR-124-2-forward: CCATTGGTGCAAACGGTCTA; miR-124-2-reverse: GTTCGACCCAGAATTTTATTTGGA; miR-124-3-forward: TTTTTTCCTGTCCTC GGCAG; miR-1243-reverse: GCCAGAGCAGAGTCTCATACCA. The primers for measuring fxr 1 transcripts were as follows: fxr1-forward1: GGCAGAAGATAGACAGC CAGT; $f x r 1$ reverse1: TTCTCCCAGAGTACGCGGTAG; fxr1-forward2: CGTCTCTTCTG TACAAGGTTCT AGG; fxr1-reverse2: TTCGGAGGGGTTAGACAGC.

Northern blot analysis. Northern blot analysis was performed as described previously (Xu et al., 2008) with some modifications. After the RNA transfer to the membrane, miRNAs were detected with $5^{\prime}$-digoxigeninlabeled mercury-LNA probes (Exiqon) specific for individual miRNAs according to the manufacturer's protocol. The membrane was then incubated with anti-digoxigenin-AP (1: 100,000 in blocking buffer) and washed multiple times. miRNAs were detected with CDP-Star substrate according to the manufacturer's protocol (Roche).

Generation of expression constructs. Flag-tagged FXR1P was cloned into the 3xFlag-pCMV7.1 vector. To generate mouse $f x r 1$-specific siRNAs, six pairs of oligos were synthesized, annealed, and cloned downstream of the $\mathrm{H} 1$ promoter into the pSuper-GFP vector (OligoEngine). The effectiveness of the siRNA constructs in HEK293T cells was determined by Western blot analysis and one was selected for further use (sense: 5'-GGCAAAGTGATCGGAAAGA-3'; antisense: $5^{\prime}$ TCTTTCCGATCACTTTGCC-3').

Cell culture and Western blot analysis. HEK293T cells were maintained in DMEM containing 10\% fetal bovine serum, and penicillin/streptomycin. Lipofectamine 2000 (Invitrogen) was used to transfect cells with expression constructs. The following primary antibodies were used: monoclonal anti-Flag M2 antibody (Sigma), anti-FMRP (Developmental Studies Hybridoma Bank, 7G1-1), anti-FMRP antibody (4317, Cell Signaling Technology), goat anti-FXR1P polyclonal antibody (Abcam, ab51970), mouse anti-FXR1P (Millipore, 6BG10); mouse anti-FXR1P antibody (4173, Cell Signaling Technology), mouse anti-FXR2P monoclonal antibody (ab37166, Abcam), and mouse anti- $\beta$-tubulin (Developmental Studies Hybridoma Bank).

Immunoprecipitation assay. HEK293 cells were transfected pSuperGFP-pre-miR-9-2 or pSuper-GFP-pre-miR-124-2 plasmids, with or without Flag-FXR1P. After $36 \mathrm{~h}$, cells were lysed using RNase-free lysis buffer (20 mм HEPES, pH 7.4, $150 \mathrm{~mm} \mathrm{NaCl}, 2 \mathrm{~mm} \mathrm{MgCl}_{2}, 0.5 \%$ Nonidet P-40, $1 \mathrm{~mm}$ dithiothreitol, and protease inhibitors). The lysates were incubated with anti-Flag antibody and protein-G-agarose beads at $4^{\circ} \mathrm{C}$ overnight. The associated RNA was isolated by regular Trizol (Invitrogen) protocol. Precipitated pre-miR-124-2 or premiR-9-2 were detected using qRT-PCR. To detect the interaction between FXR1P and Dicer, HEK293 cells were transfected with FlagFXR1P or empty vectors. Total cell lysate was prepared in $1 \%$ Triton lysis buffer after $24 \mathrm{~h}$ transfection. Two percent of the cell lysate was taken as input. The remaining cell lysate was incubated with mouse IgG or Flag antibody and subsequently with $50 \mu \mathrm{l}$ of protein G Sepharose beads. After overnight incubation at $4^{\circ} \mathrm{C}$, the beads were washed and resuspended in $75 \mu \mathrm{l}$ of sample buffer. Then, $25 \mu \mathrm{l} \mathrm{sam-}$ ples were subjected to SDS gel electrophoresis. Western blot analysis was performed using ant-Dicer and anti-FXR1P as primary antibodies.
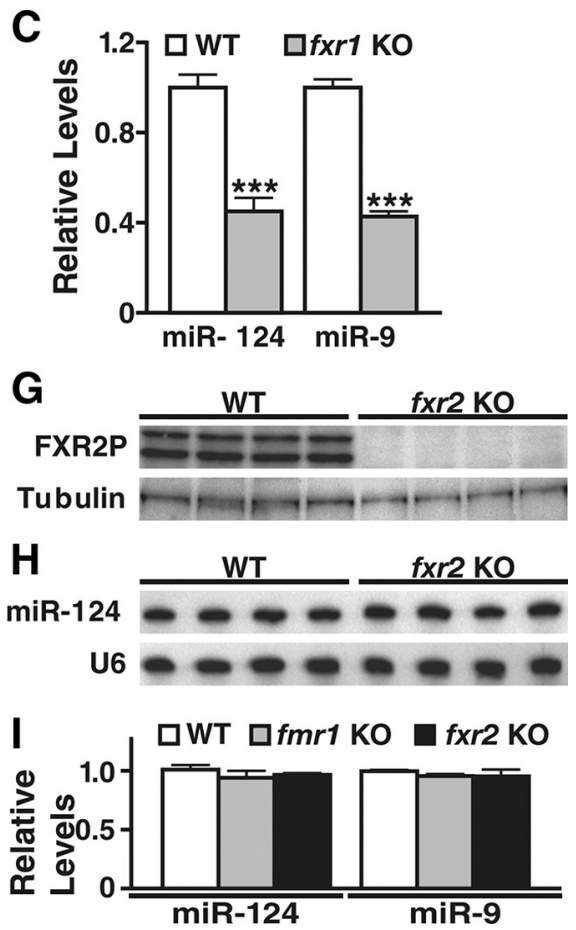

Figure 1. The levels of brain-specific miR-9 and miR-124 are regulated by FXR1P but not FMRP or FXR2P in vivo. $\boldsymbol{A}$, Brain 列 in $\boldsymbol{H}$. $\boldsymbol{H}$, Expression levels of miR-124 in the brains of four WT and four fxr $2 \mathrm{KO}$ mice at 2 months of age. I, Expression levels of miR-124 and miR-9 normalized to U6 RNA. Values are mean \pm SEM of three independent experiments.

\section{Results}

FXR1 but not FMRP is required to maintain the levels of brain-specific miR-9 and miR-124 in vivo

To determine which of the three mammalian FMRP family proteins regulates the levels of brain-specific miRNAs in vivo, especially miR-9 and miR-124, we dissected whole-brain tissues from $f x r 1$ or $f x r 2$ knock-out mice (Nelson laboratory) and fmr 1 knockout mice (Jackson Laboratory). fxr1 knock-out mice die shortly after the birth (Mientjes et al., 2004); thus, we obtained brain tissues from E18 embryos of fxr1 mutants and wild-type littermate controls. We found by Northern blot analysis that the expression of miR-124 (Fig. 1A) and miR-9 (Fig. 1B) was significantly lower in $f x r 1$ knock-out embryos (Fig. $1 C$ ), suggesting that FXR1P is required in vivo to regulate the levels of neuronal miRNAs in the brain. The level of miR-1, a muscle-specific miRNA critical for cardiovascular development (Liu and Olson, 2010), was also decreased by $\sim 45 \%$ in skeletal muscles of $f x r 1$ knock-out embryos (data not shown), indicating that the role of FXR1P is not limited to neuronal miRNAs. Misregulation of miR-1 could contribute in part to the reported muscle defects caused by FXR1P deficiency (Mientjes et al., 2004; Van't Padje et al., 2009).

FXR1P is one of the three family proteins that also include FMRP and FXR2P. To examine the effect of loss of FMRP on miRNA levels in the brain, we first confirmed the absence of FMRP in the adult brains of multiple fmr1 knock-out mice by Western blot analysis (Fig. 1D). To examine miR-9 and miR-124 

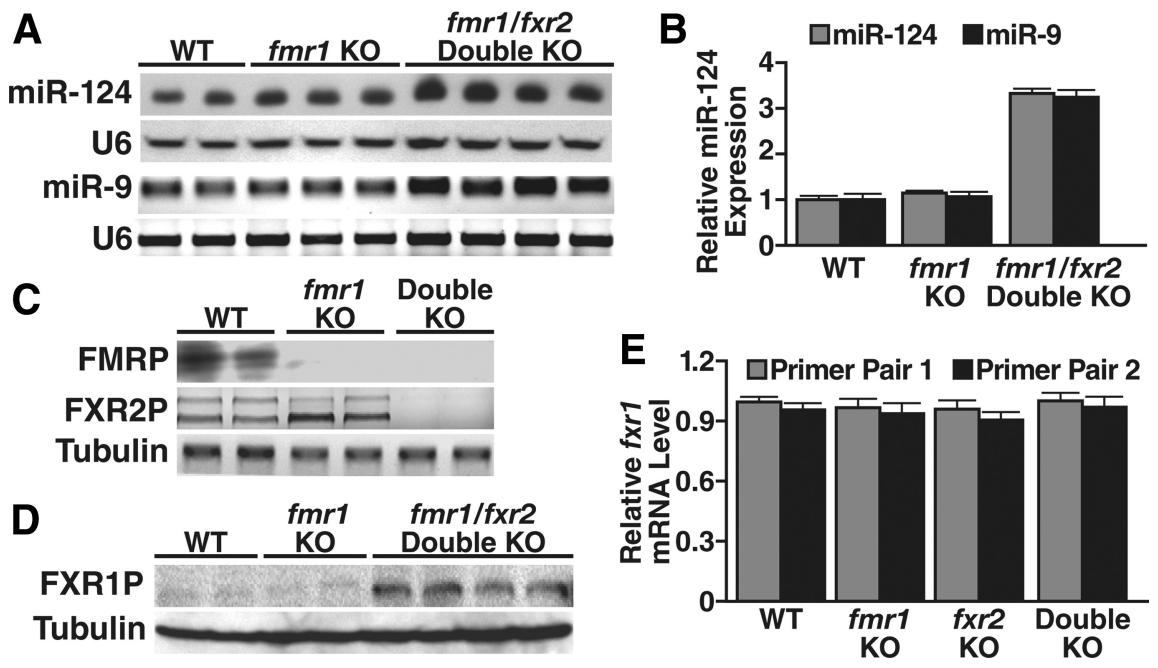

Figure 2. The levels of brain-specific miR-9 and miR-124 are elevated in $f m r 1 / f x r 2$ double-knock-out (K0) mice. $A$, The levels of miR-9 and miR-124 are significantly elevated in the brains of $f m r 1 / f x r 2$ double-K0 mice at 2 months of age. $\boldsymbol{B}$, Quantification of the levels of miR-9 and miR-124 in the brains of 2-month-old WT, fmr $1 \mathrm{KO}$, and fmr $1 / f x r 2$ double-KO mice. Values are mean \pm SEM ( $n=2-4$ mice per genotype). $p<0.001$ by Student's $t$ test for fmr $1 / f x r 2$ double-K0 mice compared with WT. The experiment was repeated with similar results. $C$, Western blot analysis confirmed the absence of Fmr1 in single-K0 mice and FMRP and FXR2P in double-KO mice. $\boldsymbol{D}$, Western blot analysis revealed a 2.4-fold increase in FXR1P expression in fmr1/fxr2 double-K0 mice $(p<$ 0.001). Equal amounts of protein lysates were loaded in each lane. $\boldsymbol{E}$, Real-time RT-PCR showed that $f x r 1 \mathrm{mRNA}$ levels remained the same in the brains of fmr 1 and fxr2 single- or double-KO mice. Values are mean \pm SEM of three independent experiments.

levels, we isolated total RNAs from whole brain tissues and performed Northern blot analysis. The level of miR-124 in the brain was not affected by loss of FMRP (Fig. 1E). This experiment was repeated three times with similar results (Fig. 1I). Similarly, miR-9 levels remained the same in fmrl knock-out mice and wild-type littermates (Fig. 1F,I). Thus, the total levels of these brain-specific miRNAs were not affected in mouse model of fragile X syndrome and are unlikely to be a contributing factor to its pathogenesis in humans.

Next, we examined the effect of loss of FXR2P, a protein that is closely related to and whose functions may overlap with those of FMRP (Zhang et al., 2008). First, we performed Western blot analysis and confirmed the absence of FXR2P in the adult brains of $f x r 2$ knock-out mice used in the following experiment (Fig. $1 G)$. Then we analyzed miR-9 and miR-124 mRNA levels in the brain by Northern blot analysis. No difference was observed between 2-month-old fxr2 knock-out mice and wild-type controls (Fig. $1 \mathrm{H}, \mathrm{I}$ ). Thus, loss of FMRP or FXR2P alone in vivo does not affect the expression levels of neuronal miRNAs in the brain.

\section{The expression levels of both FXR1P and brain-specific} miRNAs are elevated in $f m r 1 / f x r 2$ double knock-out mice FMRP and FXR2P recognize unique and common RNA targets (Darnell et al., 2009), and fmr1/fxr2 double knock-out mice exhibit more severe behavioral phenotypes (Zhang et al., 2008). To examine the effects of simultaneous loss of FMRP and FXR2P on brain-specific miRNAs, we performed Northern blot analysis of double knock-out mice. The expression level of miR-9 and miR124 (Fig. $2 A, B$ ) in the brain was significantly higher in 2-monthold fmr1/fxr2 double-knock-out mice; Western blot analysis confirmed the absence of FMRP and FXR2P (Fig. 2C). These findings suggest that FMRP and FXR2P play redundant functions in regulating miRNA levels or compensatory changes in the double knock-out mice lead to elevated levels of these brain-specific miRNAs through an indirect mechanism. Considering the importance of neuronal miRNAs in brain development and func- tion (Gao, 2010), this increase could contribute to the reported behavioral phenotypes in fmr1/fxr2 double-knockout mice (Zhang et al., 2008).

To investigate the mechanism of the effect of loss of both FMRP and FXR2P on miRNAs, we examined the expression of FXR1P, a closely related Fmr1 family protein (Zhang et al., 1995). Unexpectedly, FXR1P expression in the brains of the double-knock-out mice was 2.4-fold higher than that in wild-type mice $(p<0.001)$ (Fig. $2 D$ ) but did not seem to be significantly affected by loss of FMRP alone $(p>0.5)$ (Fig. $2 D)$. Thus, FMRP and FXR2P may act in concert to regulate FXR1P expression. This FXR1P upregulation likely occurs at the posttranscriptional level, since the fxr1 mRNA level in the brain was unaffected by simultaneous loss of FMRP and FXR2P in vivo (Fig. 2E).

\section{FXR1P modulates the processing efficiency of pre-miR-9 and pre-miR-124}

FXR1P affects miRNA-mediated translational regulation (Vasudevan et al., 2007), but its role in controlling miRNA levels is unknown. In principle, FXR1P could affect the transport, processing, or stability of premiRNAs. To distinguish these possibilities, we established an in vitro assay system in which pre-miR-124 or pre-miR-9 was ectopically expressed in HEK293 cells, which do not normally express these brain-specific miRNA. Mature miR-124 is encoded by three genes on separate chromosomes in mammals; pre-miR-124-1 and premiR-124-2 are most abundant expressed (Fig. 3A). Similarly, premiR-9-2 is the predominant gene expressed in human neural precursor cells (Delaloy et al., 2010). In HEK293 cells, transfected pre-miR-124-2 was processed (albeit inefficiently) to generate mature miR-124 (Fig. 3C). Overexpression of Flag-tagged FXR1P in HEK293 cells (Fig. 3B) increased the level of mature miR-124 in a dosedependent manner (Fig. 3C,D). Similarly, transfected pre-miR-9-2 could be processed into mature miR-9 (Fig. 3E). The apparent low processing efficiency is probably due to the high level of the transient overexpression of the miRNA precursor. Concomitant overexpression of FXR1P resulted in an increase in the level of mature miR-9 and likely a decrease in the level of the pre-miR-9-2 (Fig. 3E,F), suggesting that FXR1P promotes efficient processing of both brain-specific miRNAs.

To demonstrate a direct role for FXR1P in miRNA processing, we expressed both FXR1P and pre-miR-124-2 (or pre-miR-9-2) in HEK293 cells and performed coimmunoprecipitation experiments. qRT-PCR analysis indicates that overexpression of FXR1P decreases the level of miRNA precursors (Fig. 3G,H), consistent with the Northern blot analysis (Fig. 3C-F). Pre-miR-124-2 or pre-miR-9-2 could be found in the immunoisolates after FXR1P pull down using an Flag antibody (Fig. 3G,H), indicating that FXR1P and miRNA precursors form a common complex. Moreover, FXR1P was found to complex with Dicer, a dsRNA nuclease essential for the biogenesis of most miRNAs (Fig. 3I). FXR1P had no effect on the steady-state expression level of Dicer (Fig. 3J). Together, these findings are consistent with the notion that FXR1P affects the processing efficiency of Dicer on brain-specific miRNAs.

To further confirm the function of FXR1P in regulating miRNA levels, we also performed loss of function analysis in the 
A

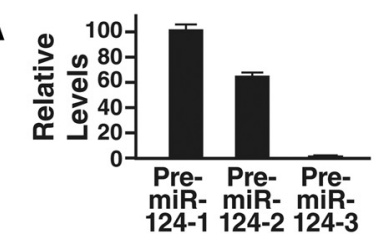

C

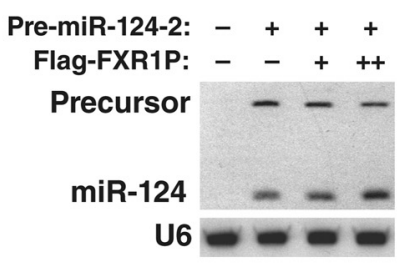

E

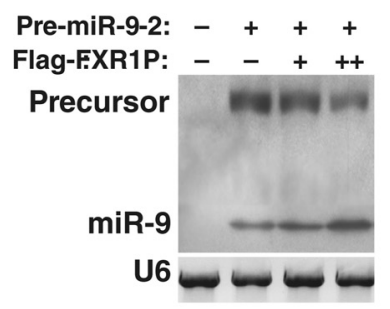

G

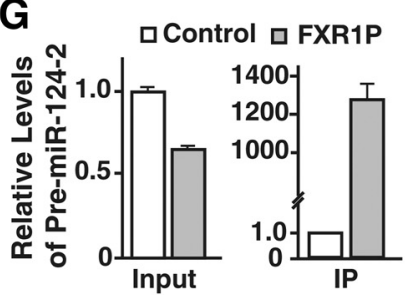

I

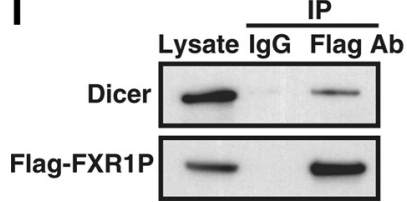

B

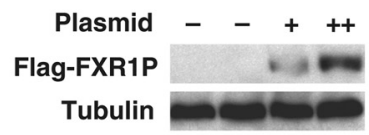

D

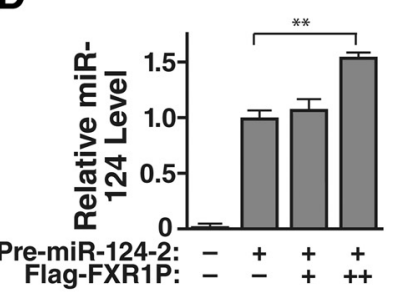

$\mathbf{F}$
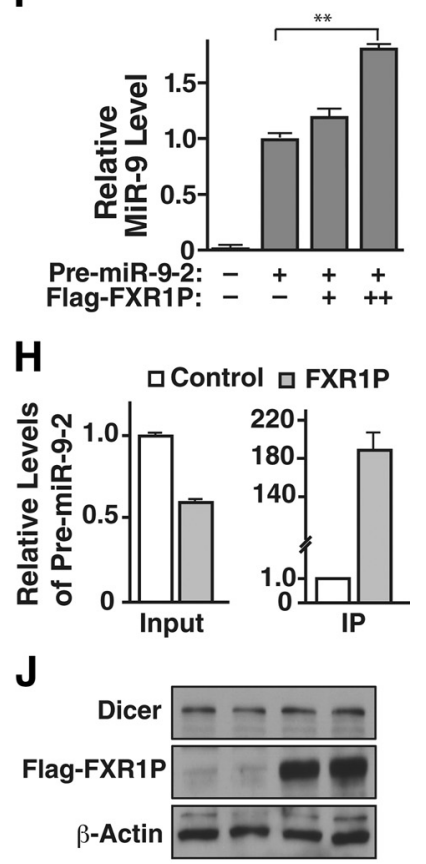

Figure 3. FXR1P affects the processing efficiency of pre-miR124 or pre-miR-9 by Dicer in HEK293 cells. $A$, The relative expression levels of three miR-124 precursors in the adult mouse brain as measured by quantitative RT-PCR. $\boldsymbol{B}$, Western blot analysis with anti-Flag antibody confirmed the ectopic expression of transfected FXR1P in HEK293 cells. C, Overexpression of Flag-FXR1P in HEK293 cells increased the steady-state level of miR-124 processed from transfected pre-miR-124-2. D, Relative miR-124 levels in transfected HEK293 cells with FXR1P overexpression. $\boldsymbol{E}$, Overexpression of Flag-FXR1P in HEK293 cells increased the steady-state level of miR-9 processed from transfected pre-miR-9-2.F, Quantification of relative miR-124 levels in transfected HEK293 cells with FXR1P overexpression. Values are mean \pm SEM of three independent experiments. ${ }^{* *} p<0.01$ by Student's $t$ test. $\boldsymbol{G}, \boldsymbol{H}$, qRT-PCR analysis indicates that pre-miR-124-2 (G) or pre-miR-9-2 (H) formed a complex with FXR1P. I, C0-IP experiment demonstrates an association between Dicer and FXR1P in HEK293 cells. IP, Immunoprecipitation with Flag antibody. J, FXR1P does not affect the steady-state level of Dicer expression as shown by Western blot analysis.

HEK293 assay system. fxr1-specific siRNAs effectively decreased the expression of FXR1P in HEK293 cells (Fig. 4D). Knockdown of endogenous FXR1P decreased the level of mature miR-124 processed from the transfected precursor molecules in HEK293 cells (Fig. $4 A, B$ ). Similarly, processing of transfected pre-miR9-2 into mature miR-9 was also compromised in the absence of endogenous FXR1P (Fig. 4B, C), consistent with out earlier finding that miR-9 or miR-124 levels were reduced in the embryonic brains of $f x r 1$ knock-out mice (Fig. 1).

A B

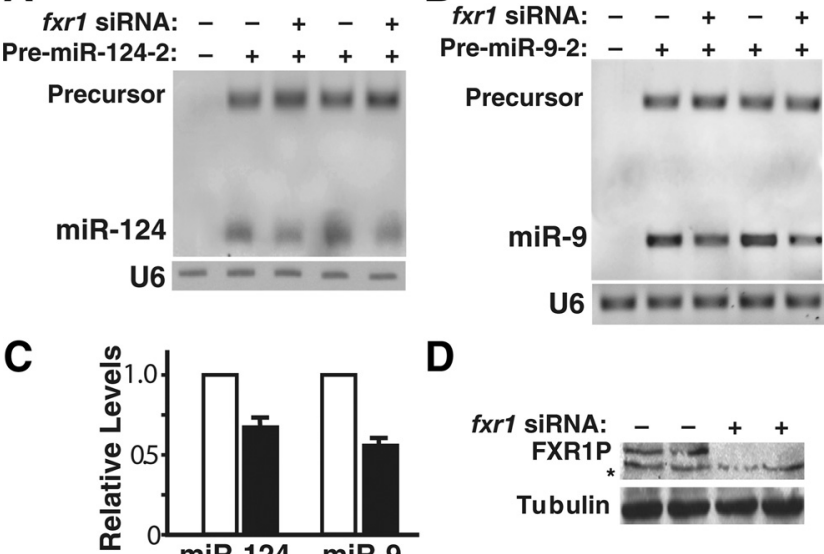

Figure 4. Loss of endogenous FXR1P affects the efficient processing of transfected brainspecific miRNA precursors. $A$, The level of mature miR-124 processed from transfected pre-miR124-2 was reduced in the absence of FXR1P. Two independent siRNA knockdown experiments are presented in this panel. $\boldsymbol{B}$, The level of mature miR-9 processed from transfected pre-miR9-2 was reduced in the absence of FXR1P. Two independent siRNA knockdown experiments are presented in this panel. $\boldsymbol{C}$, Quantification of the levels of mature miR-9 and miR-124 in the presence of absence of endogenous FXR1P.D, Fxr1 siRNA effectively decreased the expression of FXR1P in HEK293 cells, as shown by Western blot analysis. Asterisk indicates a nonspecific band recognized by the anti-FXR1P antibody. Lanes 3 and 4 are two independent knockdown experiments to show the effectiveness of the fxr 1 siRNA.

\section{Discussion}

In this study, biochemical analysis of several knock-out mouse lines revealed that loss of FMRP or FXR2P alone does not affect the expression of miR-9 and miR-124 in vivo. Surprisingly, the levels of brain-specific miR-9 and miR-124 and FXR1P were increased in fmrl/fxr2 double-knock-out mice, and FXR1P was required to maintain proper expression levels of miR-9 and miR124 in vivo. These results reveal that the total levels of these brainspecific miRNAs are regulated by FXR1P but are unchanged in a mouse model of fragile X syndrome.

In Drosophila, dFMR1 is required to maintain the proper levels of neuron-specific miR-124 (Xu et al., 2008), a function that seems to be conserved with FXR1P but not FMRP in mammals, which is consistent with comparative genomic sequence analysis indicating a closer similarity between dFMR1 and FXR proteins (Kirkpatrick et al., 2001). Such a functional divergence of mammalian homologs of fly proteins is not uncommon.

Although all three proteins are widely expressed in mammals, individual knock-out of $f m r 1$ or $f x r 2$ does not lead to gross defects in brain development or decreased survival (The Dutch-Belgian Fragile X Consortium et al., 1994; Gu et al., 2002). In contrast, fxr1 knock-out mice die shortly after the birth (Mientjes et al., 2004), suggesting an essential role for FXR1P in development. However, the precise molecular function of FXR1P is poorly understood. In human cell lines, FXR1P is involved in cell-cycledependent translational regulation through AU-rich elements and miRNAs (Vasudevan and Steitz, 2007; Vasudevan et al., 2007). Our findings reveal a role for FXR1P in controlling the abundance of neuronal miRNAs. This requirement for FXR1P is not restricted to the nervous system, since muscle-specific miR-1 is also regulated by FXR1P. Thus, the reported neuronal and muscular defects in $f x r 1$ knock-out mice could be caused in part by defects in the miRNA pathway. Moreover, fmr1/fxr2 double knock-out mice exhibited a loss of rhythmic activity in a light: dark cycle (Zhang et al., 2008), which could be at least in part 
contributed by the misregulated miRNA pathway as in flies (Kadener et al., 2009).

The expression of miRNAs can be influenced at the both transcriptional and posttranscriptional levels. Our results suggest that FXR1P is required for efficient processing of neuronal miRNAs in vivo. In DT40, a conditional Dicer knockdown cell line, FXR1P but not FMRP is increased upon Dicer knockdown and FXR1P is subject to miRNA-mediated suppression (Cheever et al., 2010). Thus, FXR1P and certain miRNAs may form a feedback loop that tightly regulates each other's expression.

Our results suggest that the levels of brain-specific miR-9 and miR-124 are not affected in a mouse model of fragile X syndrome, raising the possibility that the abundance of miRNAs, at least those examined here, does not contribute to the pathogenesis of this disorder. It remains to be determined whether the levels of other miRNAs in vivo are selectively affected by loss of FMRP alone and the extent to which misregulation of the miRNA pathway contributes to the molecular pathogenesis of fragile $\mathrm{X}$ syndrome.

\section{References}

Caudy AA, Myers M, Hannon GJ, Hammond SM (2002) Fragile X-related protein and VIG associate with the RNA interference machinery. Genes Dev 16:2491-2496.

Cheever A, Blackwell E, Ceman S (2010) Fragile X protein family member FXR1P is regulated by microRNAs. RNA 16:1530-1539.

Coolen M, Bally-Cuif L (2009) MicroRNAs in brain development and physiology. Curr Opin Neurobiol 19:461-470.

Darnell JC, Fraser CE, Mostovetsky O, Darnell RB (2009) Discrimination of common and unique RNA-binding activities among Fragile X mental retardation protein paralogs. Hum Mol Genet 18:3164-3177.

Delaloy C, Liu L, Lee JA, Su H, Shen F, Yang GY, Young WL, Ivey KN, Gao FB (2010) MicroRNA-9 coordinates proliferation and migration of human embryonic stem cell-derived neural progenitors. Cell Stem Cell 6:323-335.

Deo M, Yu JY, Chung KH, Tippens M, Turner DL (2006) Detection of mammalian microRNA expression by in situ hybridization with RNA oligonucleotides. Dev Dyn 235:2538-2548.

Edbauer D, Neilson JR, Foster KA, Wang CF, Seeburg DP, Batterton MN, Tada T, Dolan BM, Sharp PA, Sheng M (2010) Regulation of synaptic structure and function by FMRP-associated microRNAs miR-125b and miR-132. Neuron 65:373-384.

Gao F-B (2010) Context-dependent functions of specific microRNAs in neuronal development. Neural Dev 5:25.

Gregory RI, Yan KP, Amuthan G, Chendrimada T, Doratotaj B, Cooch N, Shiekhattar R (2004) The Microprocessor complex mediates the genesis of microRNAs. Nature 432:235-240.

Gu Y, McIlwain KL, Weeber EJ, Yamagata T, Xu B, Antalffy BA, Reyes C, Yuva-Paylor L, Armstrong D, Zoghbi H, Sweatt JD, Paylor R, Nelson DL (2002) Impaired conditioned fear and enhanced long-term potentiation in Fmr2 knock-out mice. J Neurosci 22:2753-2763.

Ishizuka A, Siomi MC, Siomi H (2002) A Drosophila fragile X protein interacts with components of RNAi and ribosomal proteins. Genes Dev 16:2497-2508.

Kadener S, Menet JS, Sugino K, Horwich MD, Weissbein U, Nawathean P,
Vagin VV, Zamore PD, Nelson SB, Rosbash M (2009) A role for microRNAs in the Drosophila circadian clock. Genes Dev 23:2179-2191.

Kirkpatrick LL, McIlwain KA, Nelson DL (2001) Comparative genomic sequence analysis of the FXR gene family: FMR1, FXR1, and FXR2. Genomics 78:169-177.

Krol J, Busskamp V, Markiewicz I, Stadler MB, Ribi S, Richter J, Duebel J, Bicker S, Fehling HJ, Schübeler D, Oertner TG, Schratt G, Bibel M, Roska B, Filipowicz W (2010) Characterizing light-regulated retinal microRNAs reveals rapid turnover as a common property of neuronal microRNAs. Cell 141:618-631.

Liu N, Olson EN (2010) MicroRNA regulatory networks in cardiovascular development. Dev Cell 18:510-525.

Michlewski G, Guil S, Semple CA, Cáceres JF (2008) Posttranscriptional regulation of miRNAs harboring conserved terminal loops. Mol Cell 32:383-393.

Mientjes EJ, Willemsen R, Kirkpatrick LL, Nieuwenhuizen IM, HoogeveenWesterveld M, Verweij M, Reis S, Bardoni B, Hoogeveen AT, Oostra BA, Nelson DL (2004) Fxrl knockout mice show a striated muscle phenotype: implications for Fxrlp function in vivo. Hum Mol Genet 13:1291-1302.

Newman MA, Hammond SM (2010) Emerging paradigms of regulated microRNA processing. Genes Dev 24:1086-1092.

Schratt G (2009) Fine-tuning neural gene expression with microRNAs. Curr Opin Neurobiol 19:213-219.

Shibata M, Kurokawa D, Nakao H, Ohmura T, Aizawa S (2008) MicroRNA-9 modulates Cajal-Retzius cell differentiation by suppressing Foxg1 expression in mouse medial pallium. J Neurosci 28:10415-10421.

Tamanini F, Willemsen R, van Unen L, Bontekoe C, Galjaard H, Oostra BA, Hoogeveen AT (1997) Differential expression of FMR1, FXR1 and FXR2 proteins in human brain and testis. Hum Mol Genet 6:1315-1322.

The Dutch-Belgian Fragile X Consortium, Bakker CE, Verheij C, Willemsen R, van der Helm R, Oerlemans F, Vermey M, Bygrave A, Hoogeveen AT, Oostra BA, Reyniers E, De Boule K, D'Hooge R, Cras P, van Velzen D, Nagels G, Martin J-J, De Deyn PP, Darby JK, Willems PJ (1994) Fmr1 knockout mice: a model to study fragile $\mathrm{X}$ mental retardation. Cell 78:23-33.

Van't Padje S, Chaudhry B, Severijnen LA, van der Linde HC, Mientjes EJ, Oostra BA, Willemsen R (2009) Reduction in fragile X related 1 protein causes cardiomyopathy and muscular dystrophy in zebrafish. J Exp Biol 212:2564-2570

Vasudevan S, Steitz JA (2007) AU-rich-element-mediated upregulation of translation by FXR1 and Argonaute 2. Cell 128:1105-1118.

Vasudevan S, Tong Y, Steitz JA (2007) Switching from repression to activation: microRNAs can up-regulate translation. Science 318:1931-1934.

Waung MW, Huber KM (2009) Protein translation in synaptic plasticity: mGluR-LTD, Fragile X. Curr Opin Neurobiol 19:319-326.

Xu XL, Li Y, Wang F, Gao F-B (2008) The steady-state level of the nervous system-specific microRNA-124a is regulated by dFMR1 in Drosophila. J Neurosci 28:11883-11889.

Zhang J, Fang Z, Jud C, Vansteensel MJ, Kaasik K, Lee CC, Albrecht U, Tamanini F, Meijer JH, Oostra BA, Nelson DL (2008) Fragile X-related proteins regulate mammalian circadian behavioral rhythms. Am J Hum Genet 83:43-52.

Zhang Y, O'Connor JP, Siomi MC, Srinivasan S, Dutra A, Nussbaum RL, Dreyfuss G (1995) The fragile X mental retardation syndrome protein interacts with novel homologs FXR1 and FXR2. EMBO J 14:5358-5366. 